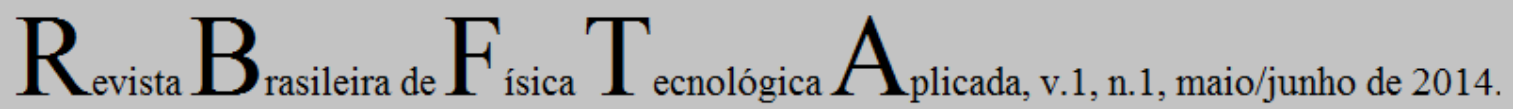

\title{
¿Los modelos mentales que enplean los alumnos sobre los conceptos de electrostática dependen de la ingeniería que estudian?
}

Os modelos mentais que os alunos aplicam sobre os conceitos de eletrostática dependem do curso de engenharia que estudam?

\section{Do the Mental Models that the Students employ on the Concepts of Electrostatics depend on the Engineering Course that they attend?}

Claudio Mario Enrique ${ }^{1}$

\begin{abstract}
Resumen: Este trabajo tiene el objeto de analizar los modelos mentales que emplean los alumnos de carreras de Ingeniería Eléctrica e Ingeniería en Sistemas de Información de la Universidad Tecnológica Nacional, Facultad Regional Santa Fe, en conceptos relacionados con la Electrostática. Para ello, se tratarán de identificar los modelos que aplican después de haber recibido los conocimientos teórico - prácticos relacionados con conceptos asociados a la Ley de Coulomb y el Campo Eléctrico., cuando cursan la materia Física II. En particular, se analizarán las cohortes 2012 y 2013. Además, se investigará si la carrera determina el grado de conocimiento sobre estos conceptos. Finalmente, y aprovechando las ventajas del Análisis Estadístico Multivariado, se propondrá una herramienta para realizar la clasificación de los alumnos para su posterior seguimiento académico personalizado.
\end{abstract}

Palabras clave: física universitaria; electrostática; modelos mentales; análisis estadístico.

Resumo: Este trabalho tem por objetivo analisar os modelos mentais que os alunos de carreiras de Engenharia Elétrica e Engenharia em Sistemas de Informação da Universidade Tecnológica Nacional, Faculdade Regional Santa Fé, aplicam em conceitos relacionados com a Eletrostática. Para isso, serão identificados os modelos que os alunos aplicam após ter recebido os conhecimentos teórico - práticos relacionados com conceitos associados à Lei de Coulomb e o Campo Elétrico, quando cursam a matéria de Física II. Em particular, serão analisadas as turmas dos anos 2012 e 2013. Também verificar-se-á se a modalidade do curso determina o grau de conhecimento sobre estes conceitos. Finalmente, utilizando as aplicações da Análise Estatística Multivariada, propõe-se uma ferramenta para realizar a classificação dos alunos a fim de verificar o seu andamento individual no decorrer do curso .

Palabras-chave: física universitária;.eletrostática; modelos mentais; análise estatística.

Abstract: The objective of this work is to analyze the mental models that students from Electrical Engineering (IE) and Engineering in Information Systems (ISI) of the National Technological University, Regional Faculty of Santa Fe employ, in concepts related to Electrostatics. In order to do so, the models which the students employ after having received the theoretical-practical knowledge, related to concepts associated with Coulomb's Law and the Electrical Field when they attend Physics II subject, will be identified. Particularly, the classes from 2012 and 2013 will be analyzed by the group. In addition, it will be investigated if the course determines the students' degree of knowledge on these concepts. Finally, by employing Statistical Multivaried Analysis, one will propose a tool to accomplish the classification of the students, in order to verify their personal academic follow-up.

Keywords: university physics; electrostatic; mental models; statistical analysis.

\footnotetext{
${ }^{1}$ Jefe del Laboratorio de Física - Profesor Adjunto - UDB Física - Departamento de Materias Básicas - Facultad Regional Santa Fe- Universidad Tecnológica Nacional - Grupo de Investigación en Enseñanza de la Ingeniería (GIEDI). Lavaisse 610, 3000 Santa Fe- Argentina. E-mail: cenriquear@yahoo.com.ar
}

RBFTA, v.1, n.1, maio/junho de 2014, p.9 


\section{Introducción}

"Los modelos mentales son construcciones internas, formadas por leyes, pautas y secuencias lógicas que los estudiantes usan para interpretar la realidad" (GRECA y MOREIRA, 2001 apud BOHIGAS y PERIAGO, 2010, p.2). El modelo mental construido por el alumno le permite dar una explicación coherente, de acuerdo a sus puntos de vista, a los fenómenos. Este proceso de construcción de modelos internos se produce por la observación y el estudio de la realidad y es de carácter abierto, dado que evoluciona con el tiempo y por lo tanto pueden ser modificables a partir de nuevas experiencias e información. La adaptación de los nuevos conceptos e ideas requiere una reestructuración de los conocimientos previos en el proceso de modelización, por lo que el tiempo empleado en esta modificación depende de la complejidad del modelo, de los conocimientos previos, y de la coherencia entre el modelo y la realidad.

Frente a una nueva situación, el alumno "pone a prueba" sus modelos mentales: si éstos les responden de manera convincente con la realidad, quedarán reforzados. Caso contrario, puede generar la necesidad de modificarlo. Por lo tanto, los modelos mentales están estrechamente relacionados con las ideas alternativas de los estudiantes. Ya se ha comprobado que en muy pocas ocasiones la exposición de las ideas científicas "correctas" produce en los alumnos el abandono de sus esquemas previos. "Éstos suelen permanecer sin cambios luego de varios años de enseñanza, e inclusive pueden convivir con las ideas científicas.

Ocurre así incluso después de haber recibido una formación basada en programas específicos para modificar las ideas previas" (FURIÓ y GUISASOLA, 2003 apud BOHIGAS y PERIAGO, 2010, p.3). En consecuencia, los errores conceptuales no deben ser considerados por una falta de conocimientos tal como habitualmente se hace, sino por la presencia de modelos mentales inadecuados. Este trabajo tiene el objeto de analizar los modelos mentales que emplean los alumnos de carreras de Ingeniería en conceptos relacionados con la Electrostática.

\section{Modelos mentales en la Física}

La Física en general es una disciplina que, en su enseñanza, no favorece la construcción de modelos mentales. Habitualmente la teoría finaliza con la expresión matemática de una ley, la tan mentada "fórmula" que buscan los alumnos y que muchas veces no permite la comprensión de un fenómeno físico que en el fondo, se matematiza mediante dicha ley.

RBFTA, v.1, n.1, maio/junho de 2014, p.10 
En general, se espera que los estudiantes de Física del nivel universitario logren explicar y predecir un fenómeno físico, tomar decisiones cuando resuelven un problema, comprender y expresarse empleando diferentes sistemas de representación, y por lo menos, transformar el conocimiento físico, aplicándolo a fenómenos estructural y conceptualmente similares. Para realizar las actividades cognitivas mencionadas, los estudiantes tienen que construir modelos mentales, cuyos productos -descripciones, explicaciones, predicciones- pueden o no, coincidir con los científicamente aceptados.

Para que el alumno avance en su aprendizaje, sus modelos mentales deben acercarse al modelo científico. Greca y Moreira (1996 y 1998), constataron que el mejor desempeño para la solución de los problemas de electromagnetismo se daba en los alumnos que habían formado un modelo mental de campo electromagnético aproximado al modelo conceptual usado por físicos expertos, mientras que los alumnos que trabajaron sólo con proposiciones (fórmulas, definiciones y enunciados de leyes) aisladas, y que se limitaron a aplicarlas mecánicamente, tuvieron menor desempeño. Para finalizar, es también importante el conocimiento previo de los sujetos: siempre los de mayor conocimiento previo tendrán más facilidad para resolver problemas, "ya que disponen de estructuras de conocimiento (esquemas) que les ponen en condición de elaborar y ejecutar los modelos mentales necesarios en los procesos cognitivos que se llevan a cabo para la resolución del problema" (SOLAZ-PORTOLÉZ Y SANJOSÉ, 2008, p.5).

\section{Estudio de caso}

Esta investigación se realizó con alumnos de las carreras Ingeniería Eléctrica (IE) e Ingeniería en Sistemas de Información (ISI) que han cursado la materia Física II en la Universidad Tecnológica Nacional, Facultad Regional Santa Fe, durante los años 2012 y 2013. Este trabajo investiga si los alumnos de IE tienen una mayor "orientación" a comprender conceptos de Electrostática que los de ISI.

Existen razones basadas en competencias que podrían influir en esta presunción: los estudiantes de IE deben necesariamente conocer estos conceptos porque son fundamentales para su carrera. Por su parte, los de ISI tienden a considerar a la Física como una especie de "contratiempo" porque, no les "va a ser útil en su profesión" asociada a la Informática. 
Si bien la materia tiene diferente duración en ambas carreras (en IE es cuatrimestral y en ISI es anual), el cuestionario fue respondido al inicio del cursado en las dos ingenierías cuando los contenidos son dados en igualdad de condiciones, excepto el profesor. En consecuencia, se pueden comparar ambos cursos casi al mismo tiempo. Independientemente de la carrera universitaria, se parte de la hipótesis de que los alumnos encuestados están entrenados en la temática que se investiga en igualdad de condiciones.

Esto se debe a que ya han trabajado con los conceptos teórico - prácticos de Electrostática, y además, han cursado y/o aprobado la materia Física I, por lo que se presume que están en condiciones de comprender leyes físicas como la de Gravitación Universal - que tiene la misma estructura que la ley de Coulomb -, .y la Tercera Ley de Newton - por tratarse en ambos casos de pares de acción y reacción -. En consecuencia, los alumnos investigados, en principio, deberían tener formados o moldeados los modelos mentales asociados a dichas leyes.

Los alumnos estudiados han sido en total 76, perteneciendo 41 a IE (54\%), y 35 a ISI (46\%). El cuestionario que se les entregó es el mismo que figura en el trabajo de Bohigas y Periago (2010) y dicha encuesta fue de carácter anónimo. El mismo consiste en cuatro preguntas donde deben usar los conceptos de la ley de Coulomb y del Campo Eléctrico y no las expresiones numéricas de dichas leyes, de modo de explicitar sus ideas y las relaciones entre ellos.

Se parte del supuesto de que "la forma en que los estudiantes piensan y razonan se refleja en lo que escriben y dibujan” (BOHIGAS y PERIAGO, 2010, p.4). Se considera excluyente el reconocer el tipo de interacción según el signo de las cargas (atracción: igual signo; repulsión: distinto signo). Finalmente, se analizarán el acercamiento de los modelos mentales de los alumnos a un modelo lógico, y dentro de éstos al aceptado por la comunidad científica.

\section{Resultados}

\subsection{Pregunta Q1}

En la Pregunta $Q_{1}$ los alumnos debían indicar cual es la fuerza de interacción electrostática entre dos esferas cargadas - consideradas puntuales - , con una con el triple de carga que la otra y del mismo signo. El objetivo fue ver si los alumnos han comprendido la simetría de dicha ley por tratarse de ser, formalmente, un par de acción - reacción. La respuesta 
correcta es la $\mathrm{N}^{\circ}$ 5. Los resultados incluyen las respuestas por carrera separada, y son los siguientes:

Tabla 1: Número de respuestas correspondientes y frecuencias relativas - entre paréntesis - para cada carrera para la pregunta $Q 1$.

\begin{tabular}{|c|c|c|c|c|c|c|}
\hline & 1 & 2 & 3 & 4 & 5 & 6 \\
\hline .E. & $0(0,0 \%)$ & $10(24,4 \%)$ & $12(29,3 \%)$ & $0(0,0 \%)$ & $19(46,3 \%)$ & $0(0,0 \%)$ \\
\hline. S.I. & $1(2,9 \%)$ & $15(42,9 \%)$ & $14(40,0 \%)$ & $2(5,7 \%)$ & $3(8,6 \%)$ & $0(0,0 \%)$ \\
\hline
\end{tabular}

De acuerdo a los resultados, puede verse que el total de los alumnos de IE han respondido de manera coherente al signo de las cargas, dado que por ser ambas positivas las fuerzas son de repulsión (opciones 2; 3; y 5). De los alumnos de ISI, 3 alumnos han respondido de manera inconsistente con este criterio (preguntas 1 y 4), por lo que se supone el desconocimiento de conceptos básicos. De todos modos, el 91,4\% ha respondido de manera coherente.

Para las respuestas elegidas de modo coherente según el signo de la carga, existen tres modelos lógicos: a. el modelo aceptado por la comunidad científica - pregunta Q1-5 -; b. el modelo "sobre la carga mayor actúa la fuerza mayor" - pregunta Q1-2 - ; c. el modelo "la fuerza mayor se debe a una mayor distribución de cargas" - pregunta Q1-3. Los resultados hallados permiten ver que el 46,3\% de los alumnos de IE han respondido de modo correcto, siendo éste el modelo más elegido, mientras que los alumnos de ISI lo han hecho en un 8,6\%. Para los alumnos de ISI prevalece el modelo $b(42,9 \%)$ seguido por el $c(40,0 \%)$; mientras que para los alumnos de IE los porcentajes de dichos modelos son $24,4 \%$ y 29,3 ; respectivamente.

\subsection{Pregunta $Q 2$}

En la pregunta Q2 se quiso investigar si los alumnos aplican correctamente la ley de Coulomb. Se les presentan dos esferas con el mismo peso pero con el doble de la carga una de la otra y del mismo signo, pendiendo de sendas cuerdas. Debido a la presencia de una repulsión electrostática, las esferas se separan. Los alumnos debieron indica cuál es la posición de equilibrio.

La respuesta correcta es la $b$, dado que por tratarse de fuerzas iguales (es un par de reacción, y se relaciona con la pregunta Q1-5), la separación relativa a la vertical para las dos esferas es la misma. Los resultados hallados son los siguientes:

RBFTA, v.1, n.1, maio/junho de 2014, p.13 
Tabla 2: Número de respuestas y frecuencias relativas para cada categoría de la pregunta Q2

\begin{tabular}{|c|c|c|c|}
\hline QQ2 & A & B & C \\
\hline I. E. & $5(12,2 \%)$ & $25(61,0 \%)$ & $11(26,8 \%)$ \\
\hline I.S.I. & $5(14,3 \%)$ & $13(37,1 \%)$ & $17(48,6 \%)$ \\
\hline
\end{tabular}

Respecto al análisis de los resultados, el 61,0\% de los alumnos de IE respondieron de manera adecuada - siendo el de mayor porcentaje -; mientras que los alumnos de ISI lo hicieron en un $37,1 \%$. La segunda opción para IE en porcentajes uso el modelo lógico de que "la fuerza mayor se debe a una mayor distribución de cargas" (26,8\%), mientras que en ISI fue la más elegida con un 48,6\%. El razonamiento de que "sobre la carga mayor actúa la fuerza mayor" fue elegida por el 12,2\% en IE, y el 14,3\% en ISI, siendo las menos elegidas en ambos cursos.

Para ver el grado de coherencia entre las respuestas obtenidas - y, en consecuencia, ver si corresponden a un modelo de razonamiento coherente -, se realizó una Tabla de Contingencia entre las preguntas Q1 y Q2. En la siguiente Tabla 3 se presentan los resultados, que se realizaron por carrera separada, y totales.

Tabla 3: Contingencia entre Q1 y Q2 para IE

\begin{tabular}{|c|c|c|c|c|c|c|}
\hline & \multicolumn{3}{|c|}{ Pregunta Q2 } & \multirow{2}{*}{ Total } \\
\hline & & & $\mathrm{a}$ & $\mathrm{b}$ & $\mathrm{c}$ & \\
\hline \multirow{15}{*}{ Pregunta Q1 } & \multirow{4}{*}{2} & Cantidad & 5 & 3 & 2 & 10 \\
\hline & & $\%$ en Pregunta Q1 & $50,0 \%$ & $30,0 \%$ & $20,0 \%$ & $100,00 \%$ \\
\hline & & $\%$ en Pregunta Q2 & $100,0 \%$ & $12,0 \%$ & $18,2 \%$ & $24,4 \%$ \\
\hline & & $\%$ en Total & $12,2 \%$ & $7,3 \%$ & $4,9 \%$ & $24,4 \%$ \\
\hline & \multirow{4}{*}{3} & Cantidad & 0 & 4 & 8 & 12 \\
\hline & & $\%$ en Pregunta Q1 & $0,0 \%$ & $33,3 \%$ & $66,7 \%$ & $100,0 \%$ \\
\hline & & $\%$ en Pregunta Q2 & $0,0 \%$ & $16,0 \%$ & $72,7 \%$ & $29,3 \%$ \\
\hline & & $\%$ en Total & $0,0 \%$ & $9,80 \%$ & $19,5 \%$ & $29,3 \%$ \\
\hline & \multirow{3}{*}{5} & Cantidad & 0 & 18 & 1 & 19 \\
\hline & & $\%$ en Pregunta Q1 & $0,0 \%$ & $94,7 \%$ & $5,3 \%$ & $100,0 \%$ \\
\hline & & $\%$ en Total & $0,0 \%$ & $43,9 \%$ & $2,4 \%$ & $46,3 \%$ \\
\hline & \multirow{4}{*}{ Total } & Cantidad & 5 & 25 & 11 & 41 \\
\hline & & $\%$ en Pregunta Q1 & $12,2 \%$ & $61,0 \%$ & $26,8 \%$ & $100,0 \%$ \\
\hline & & $\%$ en Pregunta Q2 & $100,0 \%$ & $100,0 \%$ & $100,0 \%$ & $100,0 \%$ \\
\hline & & $\%$ en Total & $12,2 \%$ & $61,0 \%$ & $26,8 \%$ & $100,0 \%$ \\
\hline
\end{tabular}

Las respuestas correctas (Q1-5 y Q2-b) fue respondida por el 43,9\% de los alumnos de IE. Los que presentaron coherencia con el modelo "sobre la carga mayor actúa la fuerza mayor" (Q1RBFTA, v.1, n.1, maio/junho de 2014, p.14 
2 y Q2-a) fue solo el 12,2\%; mientras que el modelo "la fuerza mayor se debe a una mayor distribución de cargas" (Q1-3 y Q2-c) representa el 19,5\%.

Al analizar la tabla de contingencia para Q1 y Q2 para ISI, se hallaron los siguientes resultados: el modelo científico correcto fue respondido por el 23,1\%; el modelo "sobre la carga mayor actúa la fuerza mayor" fue del 33,3\%, y el modelo: "la fuerza mayor se debe a una mayor distribución de cargas" fue del 25,7\%.

Los resultados por carrera son los presentados en la Tabla 4:

Tabla 4: Resultados por carrera

\begin{tabular}{|c|c|c|c|c|}
\cline { 2 - 5 } \multicolumn{1}{c|}{} & $\begin{array}{c}\text { Modelo científico } \\
\text { correcto }\end{array}$ & $\begin{array}{c}\text { Modelo "sobre la carga } \\
\text { mayor actúa la fuerza } \\
\text { mayor" }\end{array}$ & $\begin{array}{c}\text { Modelo: "la fuerza mayor se } \\
\text { debe a una mayor } \\
\text { distribución de cargas" }\end{array}$ & Totales \\
\hline IE & $43.9 \%$ & $12.2 \%$ & $19.5 \%$ & $75,6 \%$ \\
\hline ISI & $23,1 \%$ & $33.3 \%$ & $25.7 \%$ & $82.1 \%$ \\
\hline
\end{tabular}

Los resultados evidencian que en los alumnos de IE el modelo científicamente aprobado es el de mayor porcentaje, mientras que en los alumnos de ISI éste es menor. La relación se invierte respecto al modelo "sobre la carga mayor actúa la fuerza mayor". De todos modos, los alumnos de ISI tienen mayor porcentaje en lo que hace a respuestas de modelos lógicos.

Los resultados muestran que, para las dos carreras estudiadas, los modelos empleados por los alumnos son los siguientes: modelo científico correcto: $27,6 \%$; modelo "sobre la carga mayor actúa la fuerza mayor": 13,2\%; modelo: "la fuerza mayor se debe a una mayor distribución de cargas": $22,4 \%$.

Con el objeto de evaluar si las diferencias entre las frecuencias para cada combinación de respuestas son reales o se deben a una variación aleatoria, se realizaron los test chi - cuadrado, planteando como hipótesis nula $\mathrm{H}_{0}$ que existe independencia entre las variables - las variaciones son aleatorias -, y como hipótesis alternativa $\mathrm{H}_{1}$ que no lo son, con un nivel de significación del 5\% (0,05). Estos valores se presentan en tablas: Tabla 5, Tabla 6, Tabla 9 y Tabla 10.

Tabla 5: Q1 y Q2

\begin{tabular}{|c|c|c|c|}
\hline Estadístico & valor & gl & p \\
\hline Chi Cuadrado Pearson & 46,955 & 8 & 0.000 \\
\hline
\end{tabular}

Como el nivel de significación es inferior a 0,05, se puede rechazar $\mathrm{H}_{0}$. En consecuencia, se puede decir que las diferencias entre las respuestas no son aleatorias, o que existe una alta RBFTA, v.1, n.1, maio/junho de 2014, p.15 
correlación entre las respuestas Q1 y Q2. Los resultados obtenidos para los alumnos de IE e ISI por separado respectivamente son los siguientes - con las mismas hipótesis -:

Tabla 6: Q1 y Q2 en las dos carreras

\begin{tabular}{|c|c|c|c|}
\hline Estadístico & valor & gl & p \\
\hline Chi Cuadrado Pearson (IE) & 32,695 & 4 & 0.000 \\
\hline Chi Cuadrado Pearson (ISI) & 13,710 & 8 & 0.090 \\
\hline
\end{tabular}

En consecuencia, se rechaza la $\mathrm{H}_{0}$ para IE, mientras que se acepta para ISI.

\section{El campo eléctrico}

\subsection{Pregunta Q3}

Esta pregunta se propuso con el objeto de comprobar si los alumnos investigados comprenden el concepto de Campo Eléctrico como una característica de la distribución de carga fuente del campo, o como una interacción entre las cargas actuantes. Se les pidió en la misma que dibujen el vector Campo Eléctrico creado por una carga puntual Q en un punto del espacio en tres situaciones diferentes: con la presencia de una carga positiva; con una carga negativa; y sin carga en el punto. En los tres casos el vector Campo Eléctrico es el mismo, porque la carga Q no se modifica, ni tampoco la posición del punto en el espacio - independientemente del tipo de carga (o no) presente.

El esquema alternativo de los estudiantes asocian la existencia del Campo Eléctrico a la presencia y tipo de carga presente en el punto del espacio; por lo que dicho vector será saliente cuando haya una carga (+); entrante si la carga es (-), y no habrá campo si no hay carga. Los resultados obtenidos permiten ver que éstos son similares en ambas carreras. Las respuestas correctas son mayoritarias en la pregunta Q3-1, dado que es la más habitual a darse en los cursos de Física II donde "se coloca una carga de prueba en el punto donde se quiere saber el valor del Campo Eléctrico. Parece que no se logran moldear los modelos mentales de los alumnos con este concepto basado en la interacción entre dos cargas dispuestas de manera diferente, lo que se evidencia con las respuestas halladas en las opciones 2 y 3 de la misma pregunta. Incluso se puede decir que las respuestas no dependen del tipo de carrera en la opción 2, dado que las frecuencias relativas son muy similares. No ocurre lo mismo en la opción c. Respecto a las tres opciones correctas, sólo fueron respondidas por cinco alumnos de IE.

RBFTA, v.1, n.1, maio/junho de 2014, p.16 


\subsection{Pregunta Q4}

En la pregunta Q4 se plantearon dos situaciones en la que se les pedía a los estudiantes que comparen, primero en la opción Q4-I (Tabla 7), el Campo Eléctrico creado por una carga en un punto a una determinada distancia donde se sitúa una carga mayor con el campo creado por esta segunda carga sobre la primera.

Tabla 7: Cantidad de respuestas correspondientes a la pregunta Q4-I por carrera (correcta: b)

\begin{tabular}{|c|c|c|c|c|c|}
\hline & \multicolumn{2}{|c|}{ Curso } & \multirow{2}{*}{ Total } \\
\hline & & & IE & ISI & \\
\hline \multirow{12}{*}{ Pregunta Q4-I } & \multirow{3}{*}{ A } & $\%$ en Pregunta Q4-I & $50,00 \%$ & $50,00 \%$ & $100,00 \%$ \\
\hline & & $\%$ en Curso & $9,80 \%$ & $11,40 \%$ & $10,50 \%$ \\
\hline & & $\%$ del Total & $5,30 \%$ & $5,30 \%$ & $10,50 \%$ \\
\hline & \multirow{3}{*}{ B } & $\%$ en Pregunta Q4-I & $54,80 \%$ & $45,20 \%$ & $100,00 \%$ \\
\hline & & $\%$ en Curso & $82,90 \%$ & $80,00 \%$ & $81,60 \%$ \\
\hline & & $\%$ del Total & $44,70 \%$ & $36,80 \%$ & $81,60 \%$ \\
\hline & \multirow{3}{*}{$\mathrm{C}$} & $\%$ en Pregunta Q4-I & $50,00 \%$ & $50,00 \%$ & $100,00 \%$ \\
\hline & & $\%$ en Curso & $7,30 \%$ & $8,60 \%$ & $7,90 \%$ \\
\hline & & $\%$ del Total & $3,90 \%$ & $3,90 \%$ & $7,90 \%$ \\
\hline & \multirow{3}{*}{ Total } & $\%$ en Pregunta Q4-I & $53,90 \%$ & $46,10 \%$ & $100,00 \%$ \\
\hline & & $\%$ en Curso & $100,00 \%$ & $100,00 \%$ & $100,00 \%$ \\
\hline & & $\%$ del Total & $53,90 \%$ & $46,10 \%$ & $100,00 \%$ \\
\hline
\end{tabular}

En la opción Q4-II se realiza la misma comparación pero con las fuerzas electrostáticas que actúa sobre cada una de las cargas. El objetivo es comparar la Fuerza Electrostática y el Campo Eléctrico creado por dos cargas distintas en dos puntos situados a la misma distancia. Los resultados se presentan a continuación.

El análisis de estas preguntas, la Tabla 7 permite ver que la pregunta Q4-I fue respondida de manera correcta (opción b) por un porcentaje alto para ambas carreras.

En la pregunta Q4-II, Tabla8, para la mayoría del curso de ISI la respuesta es la opción "b", que sostiene el modelo que "la carga mayor ejerce una fuerza mayor". No obstante, para la mayoría de los alumnos de IE, el modelo empleado es el aceptado por la comunidad científica.

Tabla 8: Cantidad de respuestas correspondientes a la pregunta Q4-II por carrera (correcta: c)

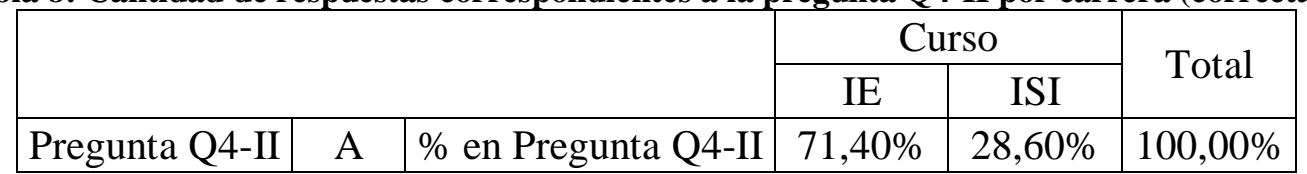

RBFTA, v.1, n.1, maio/junho de 2014, p.17 


\begin{tabular}{|c|c|c|c|c|}
\hline & $\%$ en Curso & $12,20 \%$ & $5,70 \%$ & $9,20 \%$ \\
\hline & $\%$ del Total & $6,60 \%$ & $2,60 \%$ & $9,20 \%$ \\
\hline \multirow{3}{*}{ B } & $\%$ en Pregunta Q4-II & $38,50 \%$ & $61,50 \%$ & $100,00 \%$ \\
\hline & $\%$ en Curso & $36,60 \%$ & $68,60 \%$ & $51,30 \%$ \\
\hline & $\%$ del Total & $19,70 \%$ & $31,60 \%$ & $51,30 \%$ \\
\hline \multirow{3}{*}{$\mathrm{C}$} & $\%$ en Pregunta Q4-II & $70,00 \%$ & $30,00 \%$ & $100,00 \%$ \\
\hline & $\%$ en Curso & $51,20 \%$ & $25,70 \%$ & $39,50 \%$ \\
\hline & $\%$ del Total & $27,60 \%$ & $11,80 \%$ & $39,50 \%$ \\
\hline \multirow{3}{*}{ Total } & $\%$ en Pregunta Q4-II & $53,90 \%$ & $46,10 \%$ & $100,00 \%$ \\
\hline & $\%$ en Curso & $100,00 \%$ & $100,00 \%$ & $100,00 \%$ \\
\hline & $\%$ del Total & $53,90 \%$ & $46,10 \%$ & $100,00 \%$ \\
\hline
\end{tabular}

Para comprobar el grado de razonamiento coherente, se realizaron las tablas de contingencia entre las preguntas Q4-I y Q4-II, para ambas carreras (respuestas correctas: Q4-I,b y Q4-II,c):

Los resultados obtenidos evidenciaron que el 34,2\% de los alumnos han respondido de manera correcta. Pero resulta llamativo que el 40,8 \% haya respondido con el modelo "la carga mayor ejerce una fuerza mayor". Por lo tanto, afirman que como el campo en un punto es mayor, la fuerza aplicada también será mayor, confundiendo los conceptos de que las fuerzas son interacciones entre cargas, si emplea la ley de Coulomb, o entre el Campo Eléctrico existente y la carga de prueba.

Para verificar este comportamiento, se realizó una tabla de contingencia entre las preguntas Q1 y Q4-II. El 26,3\% (20 estudiantes) respondieron de manera correcta (opciones Q1,5 y Q4-II,c). La opción Q1,3 y Q4-II,b (23,7\%) asume que "la carga mayor ejerce una fuerza mayor", y la combinación Q1,2 y Q4-II,a, admite que "sobre la carga mayor actúa una fuerza mayor" $(3,9 \%)$ ?

¿Se puede decir que los modelos mentales de los alumnos tienen asumidos los conceptos de la ley Coulomb y de Campo Eléctrico? Realizando un test Chi cuadrado para las opciones Q1 y Q4-II, se hallaron los siguientes resultados:

Tabla 9: Q1 y Q4-II

\begin{tabular}{|c|c|c|c|}
\hline Estadístico & valor & gl & p \\
\hline Chi Cuadrado Pearson & 50,339 & 8 & 0.000 \\
\hline \multicolumn{4}{|c|}{ RBFTA, v.1, n.1, maio/junho de 2014, p.18 } \\
\hline \multicolumn{4}{|c|}{. }
\end{tabular}


Como es menor que 0,05, se puede afirmar que existe correlación entre las variables. Los modelos mentales de los alumnos tienen asumidos los conceptos de la ley Coulomb y de Campo Eléctrico. Dicho de otro modo, existe un grado de correlación entre las respuestas a ambas preguntas. Realizando una tabla de contingencia ente Q2 y Q4-II se halló que el 28,9\% respondió en forma correcta (22 alumnos). El 10,5\% respondió de acuerdo al modelo "la carga mayor ejerce una fuerza mayor", y si bien es un modelo coherente, no es aceptado científicamente. Los que proponen que "la fuerza mayor se debe a una mayor distribución de cargas" tiene poca representación (3,9\%). Resulta llamativa la combinación Q2,c y Q4-II,b (23,7\%). ¿Se puede afirmar que existe un alto grado de correlación entre las variables? Realizando el test Chi cuadrado para las respuestas Q2 y Q4-II se obtuvo la siguiente tabla:

Tabla 10: Q2 y Q4-II

\begin{tabular}{|c|c|c|c|}
\hline Estadístico & valor & gl & p \\
\hline Chi Cuadrado Pearson & 17,788 & 4 & 0.019 \\
\hline
\end{tabular}

Como el coeficiente de correlación hallado $(0,019)$ es menor que 0,05 , existe un buen grado de correlación entre ambas respuestas.

\section{Propuesta de seguimiento de alumnos basados en la Estadística Multivariada}

En base a los resultados y conclusiones obtenidos anteriormente, resulta evidente que los alumnos de IE emplean modelos mentales más adecuados para los conceptos de Fuerza y Campo Electrostáticos - los aceptados por la comunidad científica - que los de ISI. Una pregunta posterior es: ¿son todos los alumnos de IE más avanzados que los de ISI? Para poder responderla, se necesita un nuevo análisis que permita identificar las similaridades - o disimilaridades - de todos los individuos investigados. Más allá de la evidencia de que los alumnos de IE moldean sus modelos mentales de manera más aproximada al modelo científicamente aceptado que los alumnos de ISI, también es cierto que no todos los alumnos de IE son más avanzados que los de ISI.

Si se desea analizar a cada alumno en particular - o agruparlos de manera independiente de la carrera universitaria - , se puede emplear el Análisis de Clústeres o Conglomerados. "El análisis clúster clasifica objetos (es decir, encuestados, productos u otras entidades) de tal forma que cada objeto es muy parecido a los que hay en el conglomerado con respecto a algún criterio RBFTA, v.1, n.1, maio/junho de 2014, p.19 
de selección predeterminado" (HAIR et al, 2005, p.492). Este conjunto de técnicas multivariadas se utilizan para clasificar a un conjunto de individuos en grupos homogéneos teniendo este análisis un marcado carácter exploratorio.

A diferencia del Análisis Discriminante - otra técnica multivariante de clasificación -, en el Análisis de clústeres los grupos son a priori desconocidos, y precisamente son los que se quieren determinar. Para este trabajo se analizaron los 76 alumnos empleando el método de análisis de conglomerados conocido como clústeres jerárquicos.

Como distancia se empleó la euclídea. Por razones de espacio, se omite la presentación de la matriz de disimilaridad, el historial de conglomeración, el dendograma y la tabla con la pertenencia de cada individuo al clúster. Sólo se van a mostrar las conclusiones arribadas con el análisis de las herramientas citadas previamente, de acuerdo a cinco clústeres:

-En el clúster 1 están los alumnos que en la pregunta Q1 usaron un modelo mental coherente, pero no aceptado por la comunidad científica (opciones 2 y 3). En las restantes preguntas han respondido acertadamente en algunas de ellas (no en todas).

-En el clúster 2 se encuentran los alumnos más avanzados. Son los que respondieron la pregunta Q1 con la opción 5 - modelo aceptado científicamente - , mientras que en las dos otras opciones respondieron de manera correcta todas; o casi todas.

-En el clúster 3 hay un alumno de ISI 2013 que si bien respondió adecuadamente las opciones de las preguntas Q2; Q4-I y Q4-II, no lo fue en la Q1.

-En el clúster 4 hay un alumno de ISI 2012 que se equivoca en todas las opciones, y además optó por 4 en la pregunta Q1 (propone fuerzas atractivas cuando las cargas son del mismo signo).

-En el clúster 5, hay un alumno de ISI 2013 que responde bien la Q4-I, pero las otras no.

En base a la pertenencia del clúster por año y carrera, se han obtenido los siguientes resultados:

Tabla 11: Cantidad de individuos por clúster.

\begin{tabular}{|c|c|c|c|c|c|}
\hline Carrera & Clúster 1 & Clúster 2 & Clúster 3 & Clúster 4 & Clúster 5 \\
\hline IE 2012 & 12 & 4 & --- & --- & --- \\
\hline IE 2013 & 10 & 15 & --- & --- & --- \\
\hline ISI 2012 & 15 & 2 & 1 & 1 & --- \\
\hline ISI 2013 & 13 & 2 & --- & --- & 5 \\
\hline
\end{tabular}


Observamos que los alumnos más avanzados están en el clúster 2, donde la mayor cantidad pertenece a IE; y en particular, del año 2013. Los alumnos que necesitarían mejorar sus modelos mentales - aunque sin mucho esfuerzo - son los del clúster 1 y del 3. Finalmente, los que necesitan mayor esfuerzo son los pertenecientes a los clústeres 4, y 5. Éstos últimos pertenecen a ISI. Esta herramienta puede tener un uso aún más interesante. Si se identifican los alumnos encuestados - no en este caso, porque la encuesta fue anónima - se puede hacer un seguimiento particular de cada uno de ellos. Si además se confeccionan varios cuestionarios asociados a cada contenido disciplinar de la materia Física II, se podría realizar una evaluación continuada de los mismos, con la opción de corregir los modelos mentales que no pertenecen a los aceptados científicamente.

\section{Conclusiones}

Este trabajo ha permitido arribar a que algunos alumnos de IE e ISI de la UTN - FRSF que cursaron la materia Física II en los años 2012 y 2013 han moldeado sus modelos mentales en conceptos de Electrostática en el marco de un modelo coherente, aunque no significa necesariamente que lo hagan dentro del modelo aceptado por la comunidad científica. Además, se ha evidenciado que existen diferencias entre los alumnos de ambas carreras, prevaleciendo los de IE. También se pudo realizar un análisis individual de los alumnos mediante la técnica

multivariante de Análisis de Clúster, tanto para analizar los modelos mentales como el desempeño del mismo, afín de poder, además, realizar un seguimiento para mejorar su rendimiento académico en el caso de que la encuesta fuese personalizada.

Este trabajo puede extenderse mediante cuestionarios a para otros conceptos de Electromagnetismo, o de Física en general. Por otro lado, resultaría interesante continuar este análisis con alumnos de Física II de otras cohortes, para disponer de mayor información para analizar. Lo que no debe hacerse es caer en el facilismo y depositar todas las responsabilidades en los alumnos. Si bien son responsables de la generación de su conocimiento, ello implica que se le faciliten las herramientas para que "moldeen" sus modelos mentales y que estén lo más cerca posible del modelo aceptado por la comunidad científica.

\section{Referencias bibliográficas}

RBFTA, v.1, n.1, maio/junho de 2014, p.21 
BOHIGAS, X. y PERIAGO, C. Modelos mentales alternativos de los alumnos de segundo curso de Ingeniería sobre la Ley de Coulomb y el Campo Eléctrico. Revista Electrónica de Investigación Educativa, 2010. Disponible en <http://redie.uabc.mx/vol12no1/contenidobohigas.html>. Consultado el 10 de agosto de 2012.

GRECA, I. M. y MOREIRA, M. A. Un estudio piloto sobre representaciones mentales, imágenes, proposiciones y modelos mentales respecto al concepto de campo electromagnético en alumnos de física general, estudiantes de posgrado y físicos profesionales. Investigações em Ensino de Ciências, 1996. Disponible en: <http://www.if.ufrgs.br/public/ensino/revista.htm>. Consultado el 1 de noviembre de 2006.

GRECA, I.M., y MOREIRA, M.A. Modelos Mentales y aprendizaje de Física en Electricidad y Magnetismo. Enseñanza de las Ciencias, v.16, n.2, p.289 a 303, 1998.

GRECA, I.M., y MOREIRA, M.A.Mental, physical and mathematical models in the teaching and learning of Physics. Science Education, v. 86, n. 1, p.106 a121, 2001.

HAIR, J. F., et al. Análisis Multivariante. $5^{\circ}$ Ed. Pearson: Madrid, 2005.

SOLAZ-PORTOLÉS, J. J. Y SANJOSÉ, V. Conocimiento previo, modelos mentales y resolución de problemas. Un estudio con alumnos de bachillerato. Revista Electrónica de Investigación Educativa, v.10, n.1, 2008. 